\title{
Strange Quark Contribution to the Nucleon Spin from Electroweak Elastic Scattering Data
}

\author{
S.F. Pate, J.P. Schaub1 \\ Physics Department, New Mexico State University, Las Cruces NM 88003, USA \\ E-mail: pate@nmsu.edu
}

\begin{abstract}
The total contribution of strange quarks to the intrinsic spin of the nucleon can be determined from a measurement of the strange-quark contribution to the nucleon's elastic axial form factor. We have studied the strangeness contribution to the elastic vector and axial form factors of the nucleon, using all available elastic electroweak scattering data. Specifically, we combine elastic $\nu p$ and $\bar{\nu} p$ scattering cross section data from the Brookhaven E734 experiment with elastic $e p$ and quasi-elastic $e d$ and $e-{ }^{4} H e$ scattering parity-violating asymmetry data from the SAMPLE, HAPPEx, G0 and PVA4 experiments. We have not only determined these form factors at individual values of momentum-transfer $\left(Q^{2}\right)$, as has been done recently, but also have fit the $Q^{2}$-dependence of these form factors using simple functional forms. We present the results of these fits using existing data, along with some expectations of how our knowledge of these form factors can be improved with data from the MicroBooNE experiment planned at Fermilab.
\end{abstract}

\section{The continuing question of the strange quark contribution to the intrinsic spin of the nucleon}

The techniques of inclusive and semi-inclusive polarized deep-inelastic scattering employed at CERN, SLAC, DESY, and Jefferson Lab have provided a wealth of information about the spin structure of the nucleon over the last 25 years. The contributions of the $u$ and $d$ quarks in the valence region have now been firmly established. As well, data from collisions of polarized protons at RHIC has done much to advance our knowledge of the limitations of the gluon spin contribution to the spin of the nucleon. The strange quark contribution to the spin of the nucleon, however, is still the subject of investigation, and the indications from deep-inelastic scattering are unclear at the moment.

Consider the determination of $\Delta s+\Delta \bar{s}$ from inclusive deep inelastic scattering combined with hyperon $\beta$-decay data; a good example would be the HERMES measurement [1] of longitudinal spin asymmetries in inclusive positron-proton and positron-deuteron deep-inelastic scattering to determine $g_{1}$ of the proton, deuteron and neutron. This measurement covered the kinematic range $0.0041<x<0.9,0.18 \mathrm{GeV}^{2}<Q^{2}<20 \mathrm{GeV}^{2}$, with the data evolved to $Q^{2}$ $=5 \mathrm{GeV}^{2}$ for analysis. Using $\mathrm{SU}(3)$ flavor symmetry, these data are combined with triplet and octet axial charges ( $F$ and $D$ from hyperon $\beta$-decay data) in a NNLO analysis to obtain the singlet axial charge and the quark contributions to the proton spin. In doing so, it is necessary

1 Present address: Department of Physics and Astronomy, Valparaiso University, Valparaiso IN 46383, USA 
to extrapolate the results into the unmeasured regions of $x$ to fill the interval $0<x<1$. The result for $\Delta s+\Delta \bar{s}$,

$$
\Delta s+\Delta \bar{s}=-0.085 \pm 0.013(\mathrm{th}) \pm 0.008(\mathrm{ex}) \pm 0.009(\mathrm{ev}) \quad \text { (HERMES inclusive) }
$$

is inconsistent with 0 to more than 4 standard deviations.

A different technique is used by the same experiment using data from semi-inclusive deepinelastic scattering, observing asymmetries in the production of charged pions from protons, and in production of charged pions and kaons from deuterons [2]. The goal of this analysis is to determine the polarized parton distribution functions $\Delta q(x)$ (and their integrals) over the measured $x$-range only; no extrapolations are performed. As a result, this analysis does not rely on $\mathrm{SU}(3)$ flavor symmetry for combination with triplet and octet axial charges. However, it is necessary to have some understanding the of the fragmentation functions which relate the fundamental lepton-quark interaction to the particles observed in the final state. The result for $\Delta s(x)$ is consistent with zero in the measured $x$-range; the integral of $\Delta s(x)$,

$$
\int_{0.023}^{0.30} \Delta s(x) d x=+0.028 \pm 0.033(\text { stat }) \pm 0.009(\text { sys }) \quad(\text { HERMES semi }- \text { inclusive })
$$

is then of course consistent with zero as well.

A similar contrasting picture is illustrated by a recent global QCD fit by de Florian, Sassot, Stratmann and Vogelsang [3] which is able to bring together the hyperon $\beta$-decay data, the inclusive and semi-inclusive deep-inelastic data from CERN, SLAC, DESY, and Jefferson Lab, and the $\vec{p} \vec{p}$ collision data from RHIC under one roof. Of special interest here is their assumption that the strange and anti-strange polarized distributions are equal; this is supported by recent COMPASS results [4]. Their functional forms allow for a node in the polarized parton distribution functions; this permits a small integral of the strangeness polarized parton distribution function if there is cancelation of positive and negative contributions from different $x$-regions. Also, their functional forms allow for SU(2) and SU(3) symmetry violation; however, the best fit does not support any significant deviation from these symmetries. They determine a "truncated first moment" of the strange quark polarized parton distribution function,

$$
\int_{0.001}^{1.0} \Delta \bar{s}(x) d x=-0.006_{-0.031}^{+0.028} \quad \text { (DSSV truncated) }
$$

where the uncertainty represents a deviation of the $\chi^{2}$ from the minimum of the fit by $2 \%$. This is clearly consistent with 0 . On the other hand, when the full $x$-range $0<x<1$ is used, the effect of SU(3) symmetry and hyperon b-decay data is seen:

$$
\int_{0}^{1.0} \Delta \bar{s}(x) d x=-0.057 \quad \text { (DSSV full) }
$$

which implies $\Delta s+\Delta \bar{s}=-0.114$. (The authors of Ref. 3] declined to quote an uncertainty for this result because of the uncertainty in the functions required for extrapolation to $x=0$.)

Additional data, of increased precision, from COMPASS at CERN on semi-inclusive deepinelastic scattering [4] only deepens the contrast. Compared to HERMES, this experiment uses a rather different polarized beam (muons instead of electrons/positrons), polarized target (solid target instead of a gas target), and detector system, but the conclusion reached is similar:

$$
\int_{0.004}^{0.3} \Delta s(x) d x=-0.01 \pm 0.01(\text { stat }) \pm 0.01(\text { sys }) \quad(\text { COMPASS semi }- \text { inclusive })
$$

$\Delta s(x)$ and its integral are consistent with zero in the measured $x$-range 2

2 See also the contribution by Roland Windmolders. 


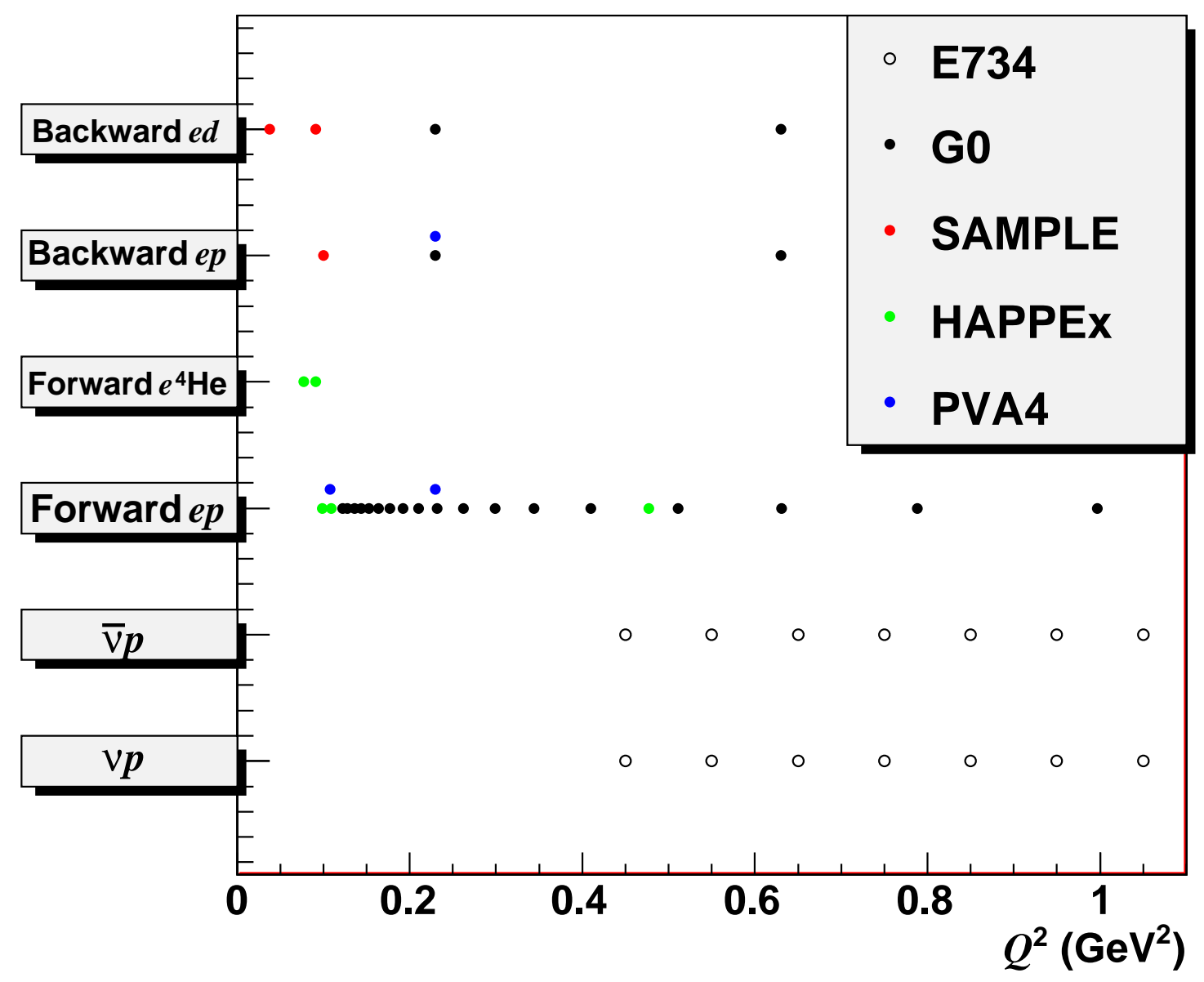

Figure 1. Overview of the types of electroweak elastic scattering data, and their $Q^{2}$ values, used in the analyses for individual values of $Q^{2}$ summarized in Figure 2, as well as in the global fit described herein. The data are from the BNL E734 [5], G0 [6, 7], SAMPLE [8], HAPPEx [9, 10, 11, 12] and PVA4 [13, 14, 15] experiments.

2. Strange quark contribution to the vector and axial form factors of the proton It is clearly of interest to examine the strange quark contribution to the nucleon spin in a way that is independent of $\mathrm{SU}(3)$ symmetry and fragmentation functions.

The full strange quark contribution to the proton spin, $\Delta S$, can be directly determined by a measurement of the strange contribution to the proton elastic axial form factor, $G_{A}^{s}$, in low energy electroweak elastic scattering.

$$
\Delta S \equiv \Delta s+\Delta \bar{s}=G_{A}^{s}\left(Q^{2}=0\right)
$$

By combining cross sections for $\nu p$ and $\bar{\nu} p$ elastic scattering with parity-violating asymmetries observed in $\vec{e} N$ elastic scattering, the strange quark contributions to the nucleon electromagnetic and axial form factors $G_{E}^{s}, G_{M}^{s}$, and $G_{A}^{s}$ may be determined simultaneously [16, 17]. The reasoning behind this method is briefly reviewed here.

In elastic scattering of polarized electrons from unpolarized nucleons, one may observe a parity-violating helicity-asymmetry in the scattering cross section, $A_{L}^{P V}$. Combining this 
information with the existing data on the nucleon vector form factors $\left(G_{E}^{p}, G_{E}^{n}, G_{M}^{p}, G_{M}^{n}\right)$, one may extract the strange quark contributions $G_{E}^{s}$ and $G_{M}^{s}$. It is assumed that charge symmetry is valid, and that the strange quark distributions in the proton and neutron are identical. This idea is the basis of the physics programs of the HAPPEx, G0, SAMPLE, and PVA4 experiments. An overview of the data from these experiments is given in Figure 1,

But the data on $A_{L}^{P V}$ are largely insensitive to the strange quark contribution to the axial form factor. To obtain information on $G_{A}^{s}$, cross sections for $\nu p$ and $\bar{\nu} p$ elastic scattering are needed; these cross sections are dominated by the axial form factor at low $Q^{2}$. The only existing data for these cross sections are from the BNL E734 experiment [5].

A number of analyses have already determined $G_{E}^{s}, G_{M}^{s}$ at individual values of $Q^{2}$, and one determines $G_{A}^{s}$ as well. These results are reviewed in Figure 2. Each different analysis has taken some subset of data covering a narrow range $Q^{2}$, and uniquely determined the form factors based on standard model expressions for the asymmetries and cross sections. The main message from these results is that the strange quark contribution to the vector form factors is consistent with zero across the full range $0.1 \mathrm{GeV}^{2}<Q^{2}<1.0 \mathrm{GeV}^{2}$. On the other hand, there is some hint of a signal of a negative $G_{A}^{s}$, but a lack of neutrino-scattering data at low $Q^{2}$ prevents a definite conclusion about $\Delta S \equiv \Delta s+\Delta \bar{s}=G_{A}^{s}\left(Q^{2}=0\right)$ at this time.
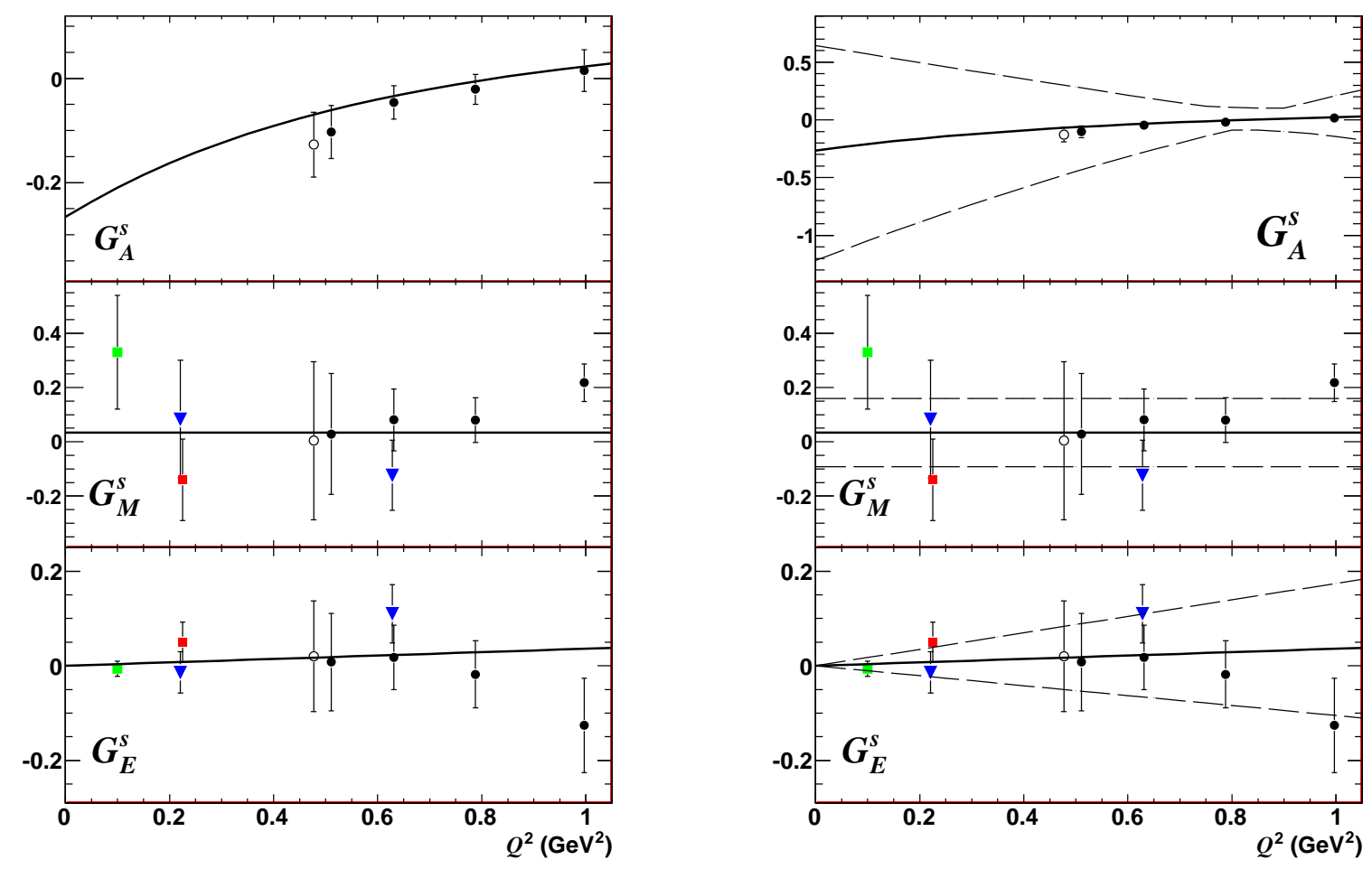

Figure 2. Results of the determination of $G_{E}^{s}, G_{M}^{s}$, and $G_{A}^{s}$ at individual values of $Q^{2}$, and also from our global fit. The separate determinations were done by Liu et al. [18] (green squares at $0.1 \mathrm{GeV}^{2}$ ), Androić et al. [7] (blue inverted triangles), Baunack et al. 15] (red squares at 0.23 $\mathrm{GeV}^{2}$ ), and Pate et al. [16] (open and closed circles). The preliminary results of the best global fit (see text) are shown by the solid line; the $70 \%$ confidence level limit curves for the fit are shown as the dashed line in the right-hand panel. The vertical scale for $G_{A}^{s}$ in the right-hand panel has been adjusted to accommodate the limit curves of the fit. 


\section{Global fit of electroweak elastic scattering data}

We have performed a global fit of the available electroweak elastic scattering data, using the same general technique described in Ref. [16], but now including all of the data in Figure 1 and assuming functional forms for the form factors. A fit of this type can quantity the amount of information that can be extracted from the electroweak data in Figure 1, provide a mechanism for including new data that may become available, and can be used as a tool to estimate the impact of any future experiments seeking to improve our knowledge of the strangeness form factors.

Our first attempt to fit the form factors $G_{E}^{s}, G_{M}^{s}$, and $G_{A}^{s}$ used this simple set of functional forms:

$$
G_{E}^{s}=\frac{\rho_{s} \tau}{\left(1+Q^{2} / \Lambda_{E}^{2}\right)^{2}} \quad G_{M}^{s}=\frac{\mu_{s}}{\left(1+Q^{2} / \Lambda_{M}^{2}\right)^{2}} \quad G_{A}^{s}=\frac{\Delta S}{\left(1+Q^{2} / \Lambda_{A}^{2}\right)^{2}} \quad \text { (initial function set) }
$$

where $\tau=Q^{2} / 4 M_{N}^{2},\left.\rho_{s} \equiv\left(d G_{E}^{s} / d \tau\right)\right|_{\tau=0}$ is the strangeness radius, $\mu_{s}$ is the strangeness magnetic moment, and the $\Lambda$ s are shape parameters that define how rapidly these functions depend on $Q^{2}$. These functions satisfy a number of boundary conditions: they all depend only on even powers of $Q$; they tend towards 0 as $Q^{2} \rightarrow \infty$, and they come to the correct physical value at $Q^{2}=0$. We found, however, that this set of functions is not quite suited to the existing data. First of all, a good fit to the neutrino data was not possible unless very large negative (and clearly unphysical) values of $\Delta S$ were permitted; this problem was solved by introducing a term linear in $Q^{2}$ in the numerator of the $G_{A}^{s}$ function. Secondly, the shape parameters $\Lambda_{E}$ and $\Lambda_{M}$ are not determined; the best fit values for them are very large, with very large errors. This is not surprising; looking at Figure 2 it seems that both $G_{E}^{s}$ and $G_{M}^{s}$ are essentially featureless, since they are consistent with zero. If $\Lambda_{E}$ and $\Lambda_{M}$ are very large, then the $Q^{2}$-dependence in the denominators of $G_{E}^{s}$ and $G_{M}^{s}$ may be neglected and we may use a simple linear function for $G_{E}^{s}$ and a constant function for $G_{M}^{s}$. Then our final set of functions were these.

$$
G_{E}^{s}=\rho_{s} \tau \quad G_{M}^{s}=\mu_{s} \quad G_{A}^{s}=\frac{\Delta S+S_{A} Q^{2}}{\left(1+Q^{2} / \Lambda_{A}^{2}\right)^{2}} \quad \text { (final function set) }
$$

The best values for the five parameters (preliminary) are:

$$
\rho_{s}=0.13 \pm 0.21 \quad \mu_{s}=0.035 \pm 0.053 \quad \Delta S=-0.27 \pm 0.41 \quad \Lambda_{A}=1.3 \pm 1.9 \quad S_{A}=0.32 \pm 0.48
$$

The best fit is shown as the solid line in Figure 2, the $70 \%$ confidence level uncertainty limits are shown by the dashed line.

It should be stressed that the fit has not been made to the values of the form factors displayed in Figure 2, Instead, the fit has been made to the measured values of the cross sections and asymmetries from the experiments mentioned in Figure 1, In Figure 2, we show that this global fit is consistent with the results of previous limited analyses of subsets of these data.

The preliminary results of the fit can be simply described. The strangeness radius and magnetic moment are consistent with zero, and the uncertainties in these parameters are consistent with the uncertainties in the separate determinations of $G_{E}^{s}$ and $G_{M}^{s}$ on display in Figure 2, On the other hand, $\Delta S$ is also consistent with 0 but the uncertainty is very large because there are no $\nu p$ or $\bar{\nu} p$ elastic data at sufficiently low $Q^{2}$ to constrain it. As a result the uncertainties in the global fit to $G_{A}^{s}$ are very much larger than the uncertainties in the separate determinations of $G_{A}^{s}$ in Figure 2

It is clear that the single greatest need is for a much improved set of data on neutral current $\nu p$ and $\bar{\nu} p$ elastic scattering down to the lowest $Q^{2}$ possible. In the last few years it has become clear that a better understanding of the charged-current cross section is also needed, as described in the next section. 


\section{The problem of the charged-current axial form factor}

The results of this fit are still preliminary because we do not have a full systematic uncertainty analysis. Such an analysis would consider variations in all the fixed input parameters, such as the full nucleon form factors, within their known limits as determined by existing experimental data or by theoretical considerations.

One nucleon form factor that must be known for this analysis to proceed is the charged-current portion of the axial form factor. The full nucleon axial form factor contains contributions from up, down, and strange quarks,

$$
G_{A}^{Z}=\frac{1}{2}\left(-G_{A}^{u}+G_{A}^{d}+G_{A}^{s}\right)
$$

and this form factor occurs in the current associated with $Z$-exchange processes like $e p, \nu p$, and $\bar{\nu} p$ elastic scattering. The "charged-current" portion of that form factor,

$$
G_{A}^{C C}=G_{A}^{u}-G_{A}^{d}
$$

occurs in $W$-exchange processes, like $\nu_{\mu}+n \rightarrow \mu^{-}+p$. The cross section for $\nu_{\mu}+n \rightarrow \mu^{-}+p$ has been studied using deuterium and nuclear targets for many years, and until a few years ago the form factor $G_{A}^{C C}$ extracted from those cross sections was considered "known." In this analysis we used the parametrization provided by Budd, Bodek and Arrington [19, 20, 21. In the analysis technique used here and in Ref. [16], it is the full axial form factor $G_{A}^{Z}$ which is determined from the neutral current $e p, \nu p$, and $\bar{\nu} p$ data; the charged-current part $G_{A}^{C C}$ must be subtacted away to obtain $G_{A}^{s}$; any uncertainty in $G_{A}^{C C}$ translates directly into an uncertainty in $G_{A}^{s}$.

In recent years, the charged-current portion of the axial form factor has become a lot less "known" than it used to be, due to high-statistics neutrino-nucleus scattering experiments such as MiniBooNE [22, 23] and $\mathrm{K} 2 \mathrm{~K}$ [24] that raise questions about our knowledge of its $Q^{2}$ dependence. Due to the imperfect knowledge of the incoming neutrino momentum and inability to reconstruct the vertex kinematics, a more complete and sophisticated way of analysing these data must be developed which takes into account the electroweak nuclear response. This is underway - see for example Benhar, Coletti, and Meloni [25], Ankowski, Behnar, and Farina [26], and references therein.

In order to improve the situation, it is necessary to have both the charged-current and neutralcurrent elastic channels measured at low $Q^{2}$ in the same experiment and treated in a consistent way to extract the axial form factor(s). The MicroBooNE experiment at Fermilab may provide these data.

MicroBooNE is an approved experiment at Fermilab to build a large liquid Argon Time Projection Chamber (LArTPC) to be exposed to the Booster neutrino beam and the NuMI beam at Fermilab. The experiment will address the low energy excess observed by the MiniBooNE experiment, measure low energy neutrino cross sections, and serve as the necessary next step in a phased program towards massive Liquid Argon TPC detectors.

Liquid argon is very well-suited to observe low energy protons from low- $Q^{2}$ neutral-current and charged-current scattering; this experiment may be an ideal place to move towards a successful determination of $\Delta s$.

\section{Summary}

The strangeness contribution to the nucleon spin may be determined from a combined analysis of low-energy electron-nucleon and neutrino-nucleon elastic scattering data.

However, existing neutral-current neutrino data (from BNL E734) lack insufficient precision and $Q^{2}$-range to make possible a definitive determination. In addition, recent neutrino 
experiments have called into question our understanding of the charged-current axial form factor extracted from data on nuclear targets.

New experiments (e.g. MicroBooNE) can provide the datasets needed for a consistent treatment of the electroweak nuclear response and extraction of the strange axial form factor.

\section{Acknowledgments}

This work was funded by the US Department of Energy, Office of Science.

\section{References}

[1] Airapetian A et al. (HERMES) 2007 Phys. Rev. D75 012007 (Preprint hep-ex/0609039)

[2] Airapetian A et al. (HERMES) 2005 Phys. Rev. D71 012003 (Preprint hep-ex/0407032)

[3] de Florian D, Sassot R, Stratmann M and Vogelsang W 2009 Phys. Rev. D80 034030 (Preprint 0904.3821)

[4] Alekseev M G et al. (COMPASS) 2010 Phys. Lett. B693 227-235 (Preprint 1007.4061)

[5] Ahrens L A et al. 1987 Phys. Rev. D35 785

[6] Armstrong D S et al. (G0) 2005 Phys. Rev. Lett. 95092001 (Preprint nucl-ex/0506021)

[7] Androić D et al. (G0) 2010 Phys. Rev. Lett. 104012001 (Preprint 0909.5107)

[8] Beise E J, Pitt M L and Spayde D T 2005 Prog. Part. Nucl. Phys. 54 289-350 (Preprint nucl-ex/0412054)

[9] Aniol K A et al. (HAPPEx) 2004 Phys. Rev. C69 065501 (Preprint nucl-ex/0402004)

[10] Aniol K A et al. (HAPPEx) 2006 Phys. Lett. B635 275-279 (Preprint nucl-ex/0506011)

[11] Aniol K A et al. (HAPPEx) 2006 Phys. Rev. Lett. 96022003 (Preprint nucl-ex/0506010)

[12] Acha A et al. (HAPPEx) 2007 Phys. Rev. Lett. 98032301 (Preprint nucl-ex/0609002)

[13] Maas F E et al. (A4) 2004 Phys. Rev. Lett. 93022002 (Preprint nucl-ex/0401019)

[14] Maas F E et al. (A4) 2005 Phys. Rev. Lett. 94152001 (Preprint nucl-ex/0412030)

[15] Baunack S et al. (A4) 2009 Phys. Rev. Lett. 102151803 (Preprint 0903.2733)

[16] Pate S F, McKee D W and Papavassiliou V 2008 Phys. Rev. C78 015207 (Preprint 0805.2889)

[17] Pate S F 2004 Phys. Rev. Lett. 92082002 (Preprint hep-ex/0310052)

[18] Liu J, McKeown R D and Ramsey-Musolf M J 2007 Phys. Rev. C76 025202 (Preprint 0706.0226)

[19] Budd H, Bodek A and Arrington J 2003 (Preprint hep-ex/0308005)

[20] Bodek A, Budd H and Arrington J 2004 AIP Conf. Proc. 698 148-152

[21] Budd H, Bodek A and Arrington J 2005 Nucl. Phys. Proc. Suppl. 139 90-95

[22] Aguilar-Arevalo A A et al. (MiniBooNE) 2008 Phys. Rev. Lett. 100032301 (Preprint 0706.0926)

[23] Aguilar-Arevalo A A et al. (MiniBooNE) 2010 Phys. Rev. D81 092005 (Preprint 1002.2680)

[24] Gran R et al. (K2K) 2006 Phys. Rev. D74 052002 (Preprint hep-ex/0603034)

[25] Benhar O, Coletti P and Meloni D 2010 Phys. Rev. Lett. 105132301 (Preprint 1006.4783)

[26] Ankowski A M, Benhar O and Farina N 2010 Phys. Rev. D82 013002 (Preprint 1001.0481) 